\title{
EFECTO DE LA SIEMBRA DIRECTA Y LABRANZA CONVENCIONAL SOBRE LA COLONIZACION MICORRIZICA Y ESPORULACION EN TRIGO
}

\author{
(Effect of no-till and conventional tillage on the mycorrhizal colonization and \\ sporulation in wheat)
}

\author{
Santiago Schalamuk ${ }^{1,3}$, Silvana Velázquez ${ }^{3}$, \\ Hugo Chidichimo ${ }^{1,2}$ \& Marta Cabello ${ }^{1,3}$ \\ ${ }^{1}$ Comisión de Investigaciones Científicas de la provincia de Buenos Aires. \\ ${ }^{2}$ Cátedra de Cerealicultura, Fac. Cs. Agrarias y Forestales, UNLP. \\ ${ }^{3}$ Instituto Spegazzini, Av. 53 No 477, B1900AVJ, \\ La Plata. E-mail: sschala@yahoo.com.ar
}

Palabras clave: Labranza, trigo, fertilización nitrogenada, colonización micorrízica. Key words: Tillage, wheat, $\mathrm{N}$ fertilization, mycorrhizal colonization.

\section{RESUMEN}

Los efectos de las prácticas agronómicas sobre las poblaciones de hongos formadores de micorrizas arbusculares han sido poco estudiados en condiciones de campo. Las comunidades de hongos micorrízicos son específicas del lugar y pueden ser afectadas de diferentes maneras por las prácticas agronómicas. Para las áreas cultivables de Argentina, la información sobre este tema es escasa.

Se compararon los efectos de siembra directa (SD), labranza convencional (LC) y fertilización nitrogenada sobre la colonización de endomicorrizas y la producción de esporas en monocultivo de trigo.

Se obtuvieron muestras de raíces en 3 estados fenológicos del cultivo: macollaje, floración y llenado de granos. Se procedió a su tinción, calculándose los porcentajes de colonización. La extracción de esporas del suelo se efectuó mediante tamizado en húmedo y decantación en gradiente de sacarosa.

La colonización se incrementó desde el macollaje hasta el llenado de granos. Sólo se observaron diferencias significativas en la colonización en macollaje en $S D$, que fue superior a $L C$.

El número de esporas en $100 \mathrm{gr}$ de suelo presentó diferencias según el sistema de labranza. En LC con fertilizante el número de esporas se duplicó desde el macollaje hasta la floración. En LC sin fertilizante este número fue bajo en el macollaje, incrementándose hasta la floración y el llenado de granos. En SD, independientemente del nivel de fertilización, el número de esporas se mantuvo estable durante todo el ciclo.

\section{ABSTRACT}

Agricultural practice effects on arbuscular mycorrhizal fungi populations have been poorly studied in field conditions. Mycorrhizal fungal communities are site specific and can be affected in different ways by agricultural practices. Information about this topic for cultivating areas in Argentina is scarce.

No-till (NT) management, conventional tillage (CT) and nitrogen fertilization effects on endomycorrhizal colonization and spore production in wheat monoculture were compared.

Root samples at three crop phenological stages: tillering, flowering and grain filling, were obtained. Then root segments were stained and the colonization percentage was calculated. Spore extraction from the soil was carried out by humid sifting and decanting in sucrose gradient.

Colonization increased from tillering to grain filling. Significant differences were only observed at the tillering stage, in which no-till management colonization was higher than conventional tillage.

Spore number in $100 \mathrm{~g}$ soil showed differences according to tillage system. In conventional tillage with a fertilizer, the spore number duplicated from tillering to flowering. In conventional tillage without a fertilizer, the spore number was low, in the tillering stage becoming higher until flowering and grain filling. In no-till management, regardless of the fertilization level, the spore number remainer stable throughout the cycle. 


\section{INTRODUCCION}

Entre los microorganismos del suelo beneficiosos para las plantas, se encuentra un grupo de hongos biótrofos que se asocian simbióticamente con las raíces para constituir un tipo de interacción conocida con el nombre de micorriza. Los hongos formadores de micorrizas arbusculares ejercen un efecto positivo sobre el crecimiento de las plantas donde los suelos son deficientes en nutrientes, particularmente fósforo. Este efecto se atribuye básicamente a: (i) mejorar la captación de nutrientes por las raíces micorrizadas, (ii) ampliar el volumen de suelo explorado, superando la zona de agotamiento de nutrientes (Cabello, 2001). El aumento en la absorción de fósforo por la colonización de hongos formadores de micorrizas arbusculares (HFMA) ha sido documentado en varias especies cultivables (Gendermann, 1968; Mosse, 1973).

En el presente, la inoculación de rutina en gran escala es dificultosa debido al alto costo de producción y el gran volumen del inóculo para el transporte (Wood, 1992). Por lo tanto, el manejo de los HFMA indígenas en el campo a través del diseño de prácticas agrícolas apropiadas puede ser efectivo con el fin de aumentar el crecimiento y la absorción de P en los cultivos. Así, los requerimientos de fertilización fosfatada se podrían reducir.

Las prácticas de labranza utilizadas para la producción de trigo en Argentina todavía están basadas en la labranza convencional, en la cual el suelo es labrado 2 o 3 veces previamente a la siembra. Sin embargo, la utilización de éstas prácticas en el largo plazo lleva a una disminución en la calidad del suelo, como se refleja en la disminución de la materia orgánica (Grace et al., 1994), pérdida de estructura (Steed et al., 1993), mayor riesgo de erosión (Packer et al., 1992) y disminución de la biota benéfica del suelo (Haines \& Uren, 1990; Abbot \& Robson, 1994; Gupta et al., 1994).

Las labranzas conservacionistas, en especial la siembra directa, constituyen una de las principales alternativas tecnológicas para contribuir a revertir la tendencia a la degradación de suelos destinados a la producción agrícola intensa y/o susceptibles a la erosión (Mulin, 2000). Una característica distintiva de los sistemas de siembra directa es la estratificación de los nutrientes poco móviles, básicamente $\mathrm{P}$ y $\mathrm{K}$, en los primeros centímetros del suelo, debido a la falta de incorporación con herramientas. Esta acumulación en superficie puede limitar su absorción por las plantas (Mallarino, 1998).

Poco es lo que se conoce acerca de los cambios de las propiedades microbiológicas del suelo con este nuevo manejo agrícola. La siembra directa modifica el ambiente suelo respecto a las labranzas convencionales, generando cambios en su biología y el desarrollo radical. Los cambios que generan los distintos sistemas de labranza en los procesos funcionales inherentes a los agroecosistemas afectarían la dinámica de colonización de estos microorganismos y la esporulación. Miller et al. (1995), documentaron un caso interesante donde el disturbio en un suelo no labrado resultó en un desarrollo reducido de los hongos formadores de micorrizas arbusculares y consecuentemente una menor absorción de fósforo en plántulas de maíz. Se ha reportado que muchos fertilizantes nitrogenados han reducido la colonización tanto en macetas como en el campo utilizando labranza convencional (Lanowska, 1966; Hayman, 1970; Chambers et al., 1980; Land et al., 1993).

La investigación sobre los hongos micorrízicos en sistemas agrícolas es valiosa tanto para determinar estrategias apropiadas de manejo como también para establecer las bases sobre las cuales se deben desarrollar las técnicas de inoculación (Sieverding, 1991).

Los efectos de las prácticas agronómicas sobre las poblaciones micorrízicas han sido poco estudiadas en condiciones de campo. Para las áreas cultivables de Argentina, la información sobre este tema es escasa.

El objetivo de éste trabajo es comparar los efectos de dos sistemas de labranza, siembra directa (SD) y labranza convencional (LC) y dos niveles de fertilización nitrogenada, sin $\mathrm{N}$ y con $90 \mathrm{~kg} / \mathrm{ha} \mathrm{N}$ sobre: (i) la dinámica de colonización de los hongos micorrízicos arbusculares; (ii) la producción de esporas, bajo 3 estados fenológicos del trigo.

\section{MATERIALES Y METODOS}

Se realizó un experimento a campo en la estación experimental “Ing. Agr. Hirshhorn”, Facultad de Ciencias Agrarias y Forestales (La Plata: Lat.: 34 59' S, Long.: $\left.57^{\circ} 59^{\prime}\right)$. El suelo responde a las características de un Argiudol típico con ligeras limitaciones en el drenaje interno.

En 2001, los análisis químicos de suelo a una profundidad de $20 \mathrm{~cm}$ mostraron la siguiente composición: 3.9-4.1\% de materia orgánica, 0.179-0.191\% de N, 10.1-11.3 ppm de P. El cultivo sembrado ese año fue trigo y el estudio a campo realizado estuvo compuesto de dos tratamientos de labranza: siembra directa (SD) y labranza convencional (LC), arado en otoño y disqueado antes de la siembra. La fertilización nitrogenada fue evaluada teniendo en cuenta la ubicación de las parcelas de fertilización en los años anteriores. Los tratamientos de fertilización fueron: testigo sin fertilización nitrogenada (N0) y $90 \mathrm{~kg} / \mathrm{ha} \mathrm{N}$ a la siembra (N90). Se utilizó urea como fertilizante nitrogenado y se aplicó al voléo. Además se adicionó Superfosfato Triple (100 kg/ha) como 
fertilizante fostatado al momento de la siembra. El diseño experimental fue completamente aleatorizado con 3 repeticiones. Para estudiar la colonización, se colectaron muestras de raíces usando un muestreo compuesto al azar. La muestra de cada uno de los bloques estuvo constituida por 5-6 submuestras tomadas en un cuadrado de 3 $\mathrm{m}^{2}$. Se tomaron muestras en tres estados fenológicos diferentes: macollaje, floración y llenado de granos. Para determinar la colonización de HFMA, las raíces fueron teñidas (Phillips \& Hayman, 1970), calculándose los porcentajes de colonización de acuerdo con la metodología propuesta por Giovannetti \& Mosse (1980). Se procesaron 3 muestras por tratamiento y de cada una de ellas se observaron 200 segmentos elegidos al azar. La extracción de esporas de suelo se efectuó mediante tamizado húmedo y decantado, siguiendo la metodología propuesta por Gendermann \& Nicolson (1963) y centrifugado en gradiente de sacarosa (Walker et al., 1982). Cada muestra se correspondió con los tres bloques que integraban cada tratamiento y los datos surgen al promediar las 3 observaciones.

Los niveles de colonización se analizaron estadísticamente (ANOVA + LSD P<0.05). Los datos del número de esporas fueron normalizados utilizando la fórmula log $(\mathrm{X}+1)$ para luego ser analizados estadísticamente utilizando ANOVA, y las medias fueron separadas utilizando el test de LSD en Statgraphics plus version 3.0.

\section{RESULTADOS Y DISCUSION}

La colonización se incrementó desde el macollaje hasta la etapa de llenado de granos (Figura 1).

Entre los distintos tratamientos sólo se registraron diferencias significativas en el estado de macollaje, donde los valores de colonización en SD fueron superiores a los de LC. No se observaron diferencias en el porcentaje de colonización entre los tratamientos fertilizados y no fertilizados.

El número de esporas en 100 gr de suelo seco (Figura 2), presentó dinámicas diferentes según el sistema de labranza. En LC con fertilizante el número de esporas se duplicó desde el estado de macollaje hasta la floración y se mantuvo relativamente estable hasta el llenado de grano. En cambio, en LC sin fertilizante se partió de un nivel muy bajo de esporas en el macollaje que luego se incremento ampliamente hasta la floración y el llenado de granos.

En SD, independientemente del nivel de fertilización, el número de esporas se mantuvo estable durante todo el ciclo del cultivo. Entre los tratamientos sólo se observaron diferencias significativas en el número de esporas en estado de macollaje, registrándose un mayor

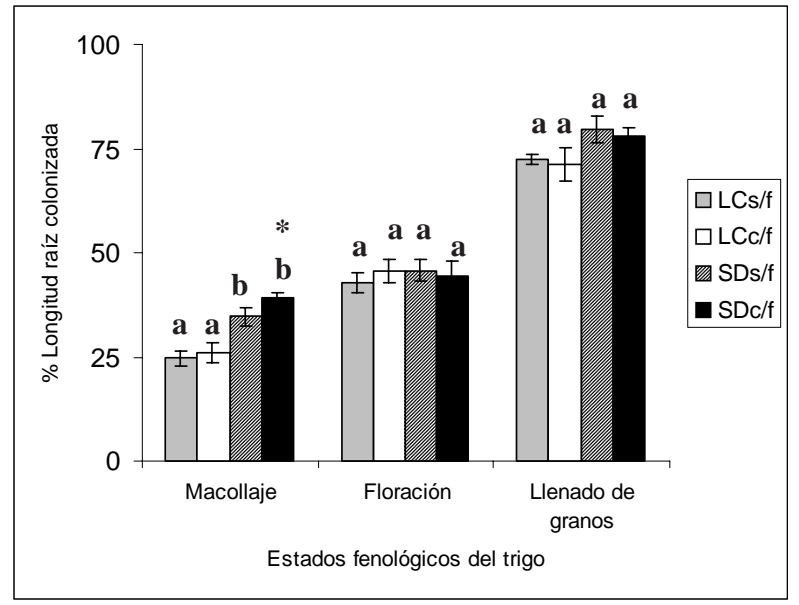

Figura 1.- Porcentaje de raíz colonizada en distintos estados fenológicos del trigo, en los distintos tratamientos.

(LCs/f: labranza convencional sin fertilizante nitrogenado; LCc/f: labranza convencional con fertilizante nitrogenado; SDs/f: siembra directa sin fertilizante nitrogenado; SDc/f: siembra directa con fertilizante nitrogenado). Diferentes letras en las columnas muestran diferencias significativas (LSD $<0.05$; * diferencia significativa LSD < 0.01). Los datos son promedios de 3 repeticiones (bloques), líneas sobre las barras indican la desviación estandar.

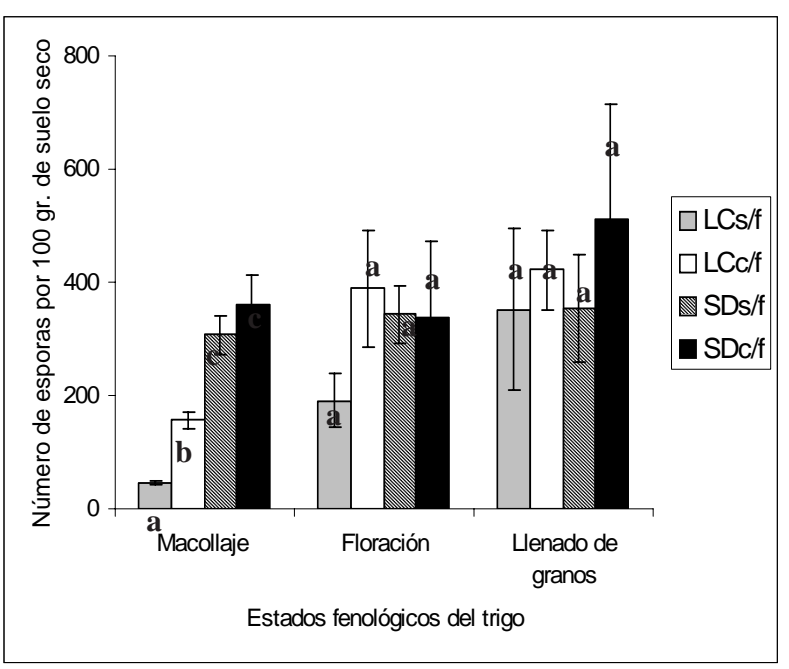

Figura 2.- Número de esporas en 100 gr de suelo seco en los distintos estados fenológicos del trigo, en los distintos tratamientos.

(Abreviaturas igual figura 1)

número en LC con fertilizante respecto a las parcelas no fertilizadas, así como también un mayor número de espodrían resultar dañadas, siendo éstas menos efectivas para producir la simbiosis (Jasper et al., 1989), afectando la colonización en los primeros estados fenológicos del cultivo. Otro efecto de la labranza es la dilución de la parte superior del suelo rica en propágulos con parte 
subsuperficial pobre en propágulos señalada por Sieverding (1991), que estaría relacionada tanto con la menor colonización en macollaje como con la menor cantidad de esporas observada.

El incremento de las esporas desde macollaje hasta llenado de granos en LC fue coincidente con los resultados de Land et al. (1993), quienes observaron en trigo bajo labranza convencional un aumento de las esporas desde 230 esporas/100 gr. en los primeros estadíos hasta 650 esporas/100 gr. al finalizar el ciclo del cultivo, un número ligeramente superior a los obtenidos en la presente investigación.

El mayor número de esporas en siembra directa, muestra la misma tendencia que los resultados obtenidos por Crovetto (1985), donde se registra un incremento en el número de esporas de 3 o 4 veces en maíz en SD con respecto a LC luego de 6 años de implementado el ensayo.

Land et al. (1993), en un suelo agrícola de Alemania, encontraron un efecto negativo, pero poco pronunciado, de la fertilización nitrogenada sobre la densidad de esporas en labranza convencional, que se registró principalmente en los últimos estados del cultivo. Sin embargo, en el presente ensayo, en labranza convencional se registró la mayor densidad de esporas en macollaje en el tratamiento fertilizado. Las diferencias encontradas podrían atribuirse a que la dosis de nitrógeno aplicado en nuestro ensayo fue notoriamente inferior.

En los estados de floración y llenado de granos no se observaron diferencias significativas en el número de esporas entre los distintos tratamientos. Estos resultados no son coincidentes con los obtenidos por Kabir et al. (1998), que observaron, en los primeros 5 $\mathrm{cm}$ de suelo, un mayor número de esporas en maíz bajo

Tabla 1. Medias y desviación estandar $(n=3)$ del porcentaje de raíz colonizada en distintos estados fenológicos del trigo, en los distintos tratamientos

\begin{tabular}{|l|c|c|c|c|c|c|}
\hline & \multicolumn{2}{|c|}{ Macollaje } & \multicolumn{2}{c|}{ Floración } & \multicolumn{2}{c|}{ Uenado de granos } \\
\hline & Media & Desvío & Media & Desvío & Media & Desvío \\
\hline LCs/f & 24,76 & $\pm 1,82$ & 42,9 & $\pm 2,5$ & 72,35 & $\pm 1,32$ \\
\hline LCclf & 26,00 & $\pm 2,32$ & 45,8 & $\pm 2,8$ & 71,29 & $\pm 3,94$ \\
\hline SDs/f & 34,63 & $\pm 2,1$ & 45,75 & $\pm 2,66$ & 79,64 & $\pm 3,22$ \\
\hline SDc/f & 39,06 & $\pm 1,19$ & 44,55 & $\pm 3,58$ & 77,99 & $\pm 2,11$ \\
\hline
\end{tabular}

(LCs/f: labranza convencional sin fertilización nitrogenada; LCc/ f: labranza convencional con fertilización nitrogenada; SDs/f: siembra directa sin fertilización nitrogenada; SDc/f: siembra directa con fertilización nitrogenada)
SD en relación con LC.

En pastizales naturales de Argentina, sin disturbios, Lugo y Cabello (2002), encontraron hasta 4083 esporas/100 ml de suelo, resultando superior al número de esporas registrado en nuestro ensayo. En este pastizal la producción de esporas mostró un patrón de distribución estacional, siendo más abundante en el otoño. En el agroecosistema de trigo estudiado, la dinámica de colonización y esporulación coincidieron con el ciclo de cultivo, lo que conjuntamente con la baja cantidad de esporas en comparación con un ecosistema natural, indica que el efecto de las prácticas agrícolas y el desarrollo del cultivo enmascara los efectos de estacionalidad.

La riqueza en especies de hongos formadores de micorrizas arbusculares encontrada en este estudio, representada por 19 especies halladas, es similar a la encontrada por Menéndez et al. (2001), quienes obtuvieron 17 especies.

Tabla 2. Medias y desviación estandar del número de esporas $(n=3)$ en 100 gr de suelo seco en los distintos estados fenológicos del trigo, en los distintos tratamientos

\begin{tabular}{|l|c|c|c|c|c|c|}
\hline & \multicolumn{2}{|c|}{ Macollaje } & \multicolumn{2}{c|}{ Floración } & \multicolumn{2}{c|}{ Uenado de granos } \\
\hline & Media & Desvío & Media & Desvío & Media & Desvío \\
\hline LCs/f & 47,00 & $\pm 3,00$ & 191,60 & $\pm 46,80$ & 352,30 & \pm 143.00 \\
\hline LCclf & 156,60 & $\pm 13,98$ & 389,30 & $\pm 104,00$ & 421,60 & $\pm 70,80$ \\
\hline SDs/f & 307,30 & $\pm 33,98$ & 343,00 & $\pm 50,00$ & 354,00 & $\pm 96,00$ \\
\hline SDclf & 359,60 & $\pm 54,11$ & 338,00 & $\pm 135,60$ & 511,00 & $\pm 204,00$ \\
\hline
\end{tabular}

(Abreviaturas igual tabla 1)

\section{CONCLUSIONES}

Las prácticas agrícolas afectan directamente los propágulos del suelo, hecho que es más evidente durante el macollaje. En el presente estudio la labranza afectó la dinámica de colonización. La falta de labranza suministró las condiciones más apropiadas para el establecimiento de la simbiosis entre las plantas y el hongo.

\section{REFERENCIAS}

Abbot, L.K. \& Robson, A.D. (1994). The impact of agricultural practices on mycorrhizal fungi. En: Pankhurst, C.E.; Doube, B.M.; Gupta, V.V.S.R. \& Grace, P.R. (eds.) Soil biota: Management in sustainable farming systems. CSIRO press, Melbourne, pp 88-95

Cabello, M.N. (2001). Mycorrhizas and hydrocarbons. En: Gadd, G.M., C.E.; Doube, B.M.; Gupta, V.V.S.R.\& Grace, P.R. (eds.) Soil biota: 
Management in sustainable farming systems. CSIRO press, Melbourne, pp 162-171

Gupta, V.V.S.R.; Roper, M.M.; Kirkegaard J.A.\& Angus J.F. (1994). Changes in microbial biomass and organic matter levels during the first year of modified tillage and stubble management practices on a red earth. Aust. J. Soil Res. 32:1339-1354

Haines, P.J. \& Uren N.C. (1990). Effects of conservation tillage farming on soil microbial biomass and organic matter and earthworm populations, in north-eastern Victoria. Aust. J. Exp. Agric. 30:365-371

Mosse, B. (1973). Advances in the study of vesicular-arbuscular mycorrhiza. Annu. Rev. Phytopathol. 11:171-196

Mulin, E. (2000). Sustentabilidad de los sistemas de siembra directa. Rev. Facultad de Agronomía. 20:285-304

Packer, I.J.; Hamilton, G.J.\& Koen, T.B. (1992). Runoff, soil loss and soil physical property changes of light textured surface soils from long-term tillage treatments. Aust. J. Soil Res. 30:789-806
Phillips, J.M. \& Hayman, D.S. (1970). Improved procedures for clearing roots and staining parasitic and VA mycorrhizal fungi for rapid assessment of infection. Trans. Br. Mycol. Soc. 55:158-161

Sieverding, E. (1991). Vesicular-Arbuscular Mycorrhiza management in Tropical Agrosystems. Deutche Gesellschaft für Technische Zusammenarbeit, GTZ N 224. Eschborn.

Steed, G.R.; Ellington, A. \& Pratley, J.E. (1993). Conservation tillage in the south-eastern Australian wheat-sheep belt. En: Carter, M.R. (ed.) Conservation tillage in temperate agroecosystems. Development and adaptation to soil and climatic constraints. Lewis, New York, pp 231-250

Wood, T. (1992). VA Mycorrhizal Fungi: Challenges for Commercialization. En: Arora, D.K.; Elander, R.P. \& Mukerji, K.G. (eds.). En: Handbook of Applied Mycology. Fungal Biotechnology. Vol. 4. Marcel Dekker, Inc. New York-Basel-Hong Kong. pp 823-848

Walker, C.; Mize, W \& McNabb, H.S. (1982). Populations of endogonaceous fungi at two populations in central Iowa. Can. J. Bot. 60: 2518-2529 